\title{
Mustguseal: versatile bioinformatic platform for knowledge-based protein design and modulation
}

\author{
Dmitry Suplatov \\ Belozersky Institute of \\ Physicochemical Biology, \\ Lomonosov Moscow State University, \\ Moscow, Russia \\ d.a.suplatov@belozersky.msu.ru
}

\author{
Yana Sharapova \\ Belozersky Institute of \\ Physicochemical Biology, \\ Lomonosov Moscow State University, \\ Moscow, Russia \\ sharapova@belozersky.msu.ru
}

\author{
Vytas Švedas \\ Belozersky Institute of \\ Physicochemical Biology and Faculty \\ of Bioengineering and Bioinformatics, \\ Lomonosov Moscow State University, \\ Moscow, Russia \\ vytas@belozersky.msu.ru
}

\begin{abstract}
Systematic bioinformatic analysis of the growing sequence and 3D-structural data representing evolutionarily distantly related proteins with diverse properties has a potential of becoming a particularly important tool in protein engineering and drug discovery, but represents a significant methodological challenge. We have developed the Mustguseal platform - a versatile on-line resource for knowledge-based protein design and modulation to facilitate experimental research, freely available at https://biokinet.belozersky.msu.ru/m-platform.
\end{abstract}

Keywords - biotechnology, bioinformatic analysis, methods on-line, protein engineering, drug discovery

\section{Motivation and aim}

\section{Motivation}

Comparative analysis of proteins implementing diverse functional properties within shared 3D-structure of the superfamily is the cornerstone of computational biology to study the relationship between structure and function and solve practical problems of medicine and biotechnology. As number of available protein sequences and 3D-structures has increased exponentially in recent years, their systematic analysis presents growing methodological and computational challenges.

Aim

The common problem of available bioinformatic tools is that they require profound skills in computational biology, and were designed to handle relatively small datasets. This impedes practical use by many investigators in a daily laboratory practice and offers limited productivity at largescale analysis of families/superfamilies. Our aim was to develop easy-to-use tools featuring content-rich interactive on-line output that will provide advanced computational methods directly to wet-lab scientists.

\section{Results}

We have developed the Mustguseal platform - a collection of easy-to-use on-line methods that provide access to comprehensive bioinformatic analysis in a daily laboratory practice. The key web-server Mustguseal can automatically collect and superimpose thousands of sequences and 3Dstructures of homologous proteins with high structure, but low sequence similarity to the selected query protein [1]. This data represent all currently known sequence variability within a common fold of a functionally diverse superfamily and can be further studied using the sister web-methods. The Zebra2 web-server can be used to identify subfamily-specific positions in protein structures, i.e. the variable amino acid residues responsible for functional diversity within the common fold, as well as the conserved positions highlighting key catalytic and structural residues. This output can be used to understand how protein/enzyme performs its inherent function, and to select hotspots for rational design or directed evolution experiments [2]. The pocketZebra web-server is an add-on to Zebra2 that maps subfamily-specific positions onto the protein surface to identify and rank binding sites by functional significance, annotate novel regulatory centers, and select particular positions in the structure that are important for selective binding of substrates/inhibitors/effectors [3]. The Yosshi web-server can systematically classify and study disulfide bonds in protein families, and assist the selection of hot-spots for disulfide engineering [4]. The visualCMAT webserver can help to select and interpret correlated mutations/coevolving residues in protein structures to reveal functionally coupled residues between topologically independent sites, study allosteric communication pathways, and select compensatory mutations for protein engineering [5]. The integration of these web-based bioinformatic tools provides an open-access out-of-the-box solution, first of its kind, to systematically analyze all the available sequence and structural data describing evolutionarily close and distantly related proteins.

We have used the developed tools to assist the experimental research in the fields of protein engineering and drug discovery - to study structure-function relationship in several protein families and design novel enzymes with improved properties (Ntn-hydrolases, penicillin-binding proteins, sialidases, $\alpha / \beta$-hydrolases, $\quad$ PLP-dependent enzymes), to identify previously unknown binding sites for modulating ligands and assist the design of complementary selective inhibitors [6-11].

We expect that our bioinformatic tools will further bridge the gap between methods of advanced computational biology and experimental studies, thus promoting the value of bioinformatics in protein engineering and drug discovery.

\section{ACKNOWLEDGMENT}

The study was funded by Russian Foundation for Basic Research according to the research project (18-29-13060). The use of HPC computing resources at the Lomonosov Moscow State University supported by the project RFMEFI62117X0011 is acknowledged [12].

\section{REFERENCES}

[1] Suplatov D., Kopylov K., Popova N., Voevodin V1., \& Švedas V. (2018) Mustguseal: a server for multiple structure-guided sequence alignment of protein families. Bioinformatics, 34(9): 1583-1585.

[2] Suplatov D., Sharapova Y., Švedas V. (2020) Zebra2: advanced and easy-to-use web-server for bioinformatic analysis of subfamilyspecific and conserved positions in diverse protein superfamilies. Nucleic acids research. doi:10.1093/nar/gkz385 
[3] Suplatov D., Kirilin E., Arbatsky M., Takhaveev V., Švedas V. (2014) pocketZebra: a web-server for automated selection and classification of subfamily-specific binding sites by bioinformatic analysis of diverse protein families. Nucleic acids research. 42(W1): 344-349.

[4] Suplatov D., Timonina D., Sharapova Y., Švedas V. (2019) Yosshi: a web-server for disulfide engineering by bioinformatic analysis of diverse protein families. Nucleic acids research. 47(W1): 308-314.

[5] Suplatov D., Sharapova Y., Timonina D., Kopylov K., Švedas V. (2018) The visualCMAT: A web-server to select and interpret correlated mutations/co-evolving residues in protein families. J. Bioinf. Comput. Biol. 16(02): 1840005

[6] Suplatov D., Besenmatter W., Švedas V., Svendsen A. (2012) Bioinformatic analysis of alpha/beta-hydrolase fold enzymes reveals subfamily-specific positions responsible for discrimination of amidase and lipase activities. Protein Eng. Des. Sel. 25(11): 689-697.

[7] Suplatov D., Panin N., Kirilin E., Shcherbakova T., Kudryavtsev P., Švedas V. (2014) Computational design of a pH stable enzyme: understanding molecular mechanism of penicillin acylase's adaptation to alkaline conditions. PloS one. 9(6).

[8] Suplatov D., Voevodin V., Švedas V. (2015) Robust enzyme design: Bioinformatic tools for improved protein stability. Biotechnology $J$. 10(3): $344-355$
[9] Fesko K., Suplatov D., Švedas V. (2018) Bioinformatic analysis of the fold type I PLP-dependent enzymes reveals determinants of reaction specificity in 1-threonine aldolase from Aeromonas jandaei. FEBS open bio. 8(6): 1013-1028.

[10] Sharapova Y., Suplatov D., Švedas V. (2018) Neuraminidase A from Streptococcus pneumoniae has a modular organization of catalytic and lectin domains separated by a flexible linker. FEBS J. 285(13):24282445.

[11] Suplatov D., Shmalgauzen E., Muronets V., Švedas V. (2018) Selective inhibitors of glyceraldehyde-3-phosphate dehydrogenase of Mycobacteria. Patent \#2661151RU.

[12] Sadovnichy V., Tikhonravov A., Voevodin V., Opanasenko V. (2013) Lomonosov: supercomputing at moscow state university. In Vetter J. (ed.) Contemporary High Performance Computing: From Petascale Toward Exascale (Chapman \& Hall/CRC Computational Science). Boca Raton, CRC Press, 283-307. 